\title{
Tai chi training reduces self-report of inattention in healthy young adults
}

\author{
Alexander K. Converse ${ }^{1}{ }^{*}$, Elizabeth O. Ahlers ${ }^{1}$, Brittany G. Travers ${ }^{1}$ and Richard J. Davidson ${ }^{1,2}$ \\ ${ }^{1}$ Waisman Center, University of Wisconsin-Madison, Madison, WI, USA \\ ${ }^{2}$ Department of Psychology, University of Wisconsin-Madison, Madison, WI, USA
}

\section{Edited by:}

Laura Schmalzl, University of

California San Diego, USA

Reviewed by:

Norman Farb, Baycrest, Canada

Peter M. Wayne, Harvard Medical

School, USA

\section{${ }^{*}$ Correspondence:}

Alexander K. Converse, Waisman

Center, University of

Wisconsin-Madison, 1500 Highland

Avenue, Room T123, Madison, WI

53705, USA

e-mail: akconverse@wisc.edu
It is important to identify effective non-pharmacological alternatives to stimulant medications that reduce symptoms of attention deficit hyperactivity disorder (ADHD). In this study of healthy young adults, we measured the effects of training in tai chi, which involves mindful attention to the body during movement. Using a non-randomized, controlled, parallel design, students in a 15-week introductory tai chi course $(n=28)$ and control participants $(n=44)$ were tested for ADHD indicators and cognitive function at three points over the course of the 15-weeks. The tai chi students' self-report of attention, but not hyperactivity-impulsivity, improved compared to controls. At baseline, inattention correlated positively with reaction time variability in an affective go/no-go task across all participants, and improvements in attention correlated with reductions in reaction time variability across the tai chi students. Affective bias changed in the tai chi students, as reaction times to positive- and negative-valenced words equalized over time. These results converge to suggest that tai chi training may help improve attention in healthy young adults. Further studies are needed to confirm these results and to evaluate tai chi as therapy for individuals with ADHD.

Keywords: Tai chi, attention deficit disorder with hyperactivity, meditation, mindfulness, non-pharmacological intervention, college students, young adults

\section{INTRODUCTION}

The use of mind-body techniques to enhance cognitive function in young adults and adolescents would provide an attractive alternative to pharmacological treatment of conditions such as attention deficit hyperactivity disorder (ADHD), as well as non-medical use of stimulants for performance enhancement (Sussman et al., 2006; Krisanaprakornkit et al., 2010). Tai chi training may provide cognitive benefits to younger individuals as it has been shown to improve cognitive function in the elderly (Matthews and Williams, 2008; Man et al., 2010; TaylorPiliae et al., 2010; Lam et al., 2012; Mortimer et al., 2012; Nguyen and Kruse, 2012; Lu etal., 2013). In this study we examine the effects of tai chi training on cognitive function in young adults.

Tai chi involves mindful attention to the body during a welldefined series of slow-flowing movements (Kauz, 1974; Jou, 1980). It is generally recognized as a safe and low-cost complementary therapy and is practiced by two million Americans for a variety of purposes (Barnes, 2004; Birdee et al., 2009). Recently, rigorous scientific methods have been applied to the study of the biomedical aspects of tai chi. While the purported health benefits of tai chi include psychological components, e.g., the cultivation of a state of relaxed attention, the majority of scientific studies focus on physical outcomes such as gait, posture, and cardiovascular health. These have largely examined middle-aged and elderly subjects (Wang et al., 2004a).

If tai chi were shown to improve attention in healthy subjects, it would provide support for future work to assess tai chi's efficacy as therapy for individuals with ADHD. ADHD affects 3-7\% of children in the U.S. and persists into adulthood in $30-70 \%$ of cases (American Psychiatric Association, 2000; Lara et al., 2009). More than three million American patients with ADHD are treated with the stimulants amphetamine and methylphenidate, both of which target the dopamine transporter (Levy, 1991; Swanson et al., 2007). Brain imaging studies also suggest the dopamine system plays a role in ADHD (Durston, 2010). Medications are in some cases ineffective, poorly tolerated, or not desired (Kooij et al., 2010; Castells et al., 2011; Green and Rabiner, 2012). Therefore, non-pharmacological therapies are the subject of research (Toplak et al., 2008; Zylowska etal., 2008; Knouse and Safren, 2010; Krisanaprakornkit et al., 2010; Young and Amarasinghe, 2010). In a non-controlled study of 13 adolescent individuals with ADHD, teacher ratings of symptoms improved following a 5-week tai chi course (Hernandez-Reif et al., 2001). Moreover, teenagers who struggle with ADHD-like symptoms but do not meet full criteria for ADHD have been shown to be at increased risk for several psychiatric disorders (Malmberg et al., 2011), suggesting that non-pharmacological interventions for inattention and hyperactivity in healthy individuals may also be warranted.

Here we report an observational study comparing healthy young adults undergoing 15 weeks of tai chi training to a passive control group. The outcome measures were cognitive function, physical balance, and ADHD indicators, all measured at the beginning, middle, and end of the semester. Because spatial working memory (SWM) and response inhibition are associated with $\mathrm{ADHD}$ and respond to stimulant therapy, our primary a priori hypothesis was that, compared to controls, subjects in the tai 
chi course would show improvements in specific measures within these neurocognitive domains (Chamberlain et al., 2011). Given reports that $\mathrm{ADHD}$ patients exhibit greater reaction time (RT) variability (Tamm et al., 2012; Kofler et al., 2013), in post hoc analyses we examined correlations between RT variability and ADHD measures.

\section{MATERIALS AND METHODS SUBJECTS}

Tai chi students were recruited from the University of WisconsinMadison course "Introduction to Martial Arts: Tai Chi" and were compensated \$30. Control subjects were recruited from the UW course "Introduction to Psychology" and were compensated with course extra credit. Subjects were required to be between the age of 18 and 34 years, and there were no exclusion criteria. Recruitment and retention details are shown in Table 1, and participant demographics are presented in Table 2. The tai chi students were older than the control subjects $(24.1 \pm 3.5$ vs. $19.4 \pm 1.3$ years $)$, but otherwise there were no significant differences. All procedures were approved by the UW Social and Behavioral Sciences Institutional Review Board (SE-2012-0539), and the study

Table 1 | Recruitment and retention.

\begin{tabular}{|c|c|c|}
\hline Number participating & Control subjects & Tai Chi students \\
\hline Session 1 & 57 & 34 \\
\hline 1 and 2 and 3 & 40 & 26 \\
\hline 1 and 2 (not 3 ) & 4 & 1 \\
\hline 1 and 3 (not 2) & 0 & 2 \\
\hline Included in analysis ${ }^{a}$ & $44(77 \%)$ & $28(82 \%)$ \\
\hline
\end{tabular}

a Participants were included in the analysis if they participated in test session 1 and at least one additional test session (2 or 3). One of the two tai chi students who participated at session 3 but not at session 2 provided incomplete ASRS data at session 3 and was therefore excluded from the analysis. Percentage indicates retention, i.e., number included in analysis/number participating in session 1.

Table 2 | Participant demographics.

\begin{tabular}{|c|c|c|c|}
\hline & Control subjects & Tai Chi students & $p^{\mathbf{e}}$ \\
\hline$n$ & 44 & 28 & \\
\hline Sex (Female) & $31(70 \%)$ & $16(57 \%)$ & 0.367 \\
\hline Age $(\text { mean } \pm S D)^{a}$ & $19.36 \pm 1.27$ & $24.14 \pm 3.46$ & $<0.001$ \\
\hline$E S L^{b}$ & $14(32 \%)$ & $6(21 \%)$ & 0.490 \\
\hline Mind-bodyc & 17 (39\%) & $13(46 \%)$ & 0.683 \\
\hline Exercise $(\text { mean } \pm S D)^{d}$ & $51.2 \pm 26.1$ & $50.8 \pm 35.6$ & 0.952 \\
\hline
\end{tabular}

a At start of semester, ${ }^{b}$ number of subjects reporting English as second language, ${ }^{c}$ number of subjects reporting previous mind-body experience (mindfulness, meditation, yoga, etc.), d Godin leisure time questionnaire Weekly Leisure Activity Score. ${ }^{e} p$ value for group difference, two sample t-test for age and exercise and chi-squared test for sex, ESL, and mind-body. was registered with ClinicalTrials.gov as a non-randomized trial (NCT01681082).

\section{INTERVENTION}

Tai chi students attended $50 \mathrm{~m}$ classes twice per week for 15 weeks, with approximately 20 students in each class. The course emphasized experiential learning with three weeks of introductory sessions on gait, posture, and tai chi principles followed by instruction in the 24-form Yang style sequence (Qu, 1999). The course has been taught for over 10 years by the same instructor, who emphasizes the mindfulness aspect of tai chi. The instructor checked attendance at each class and after a fourth absence, the final grade was lowered by one-half grade for each additional absence. Control subjects were given no training or instructions.

\section{PROTOCOL}

All subjects underwent 1-h test sessions at the beginning, middle, and end of the 15-week semester to assess balance, cognitive function, and ADHD indicators. Testing was performed on one set of tai chi students and controls during Fall 2012 and on a second set during Spring 2013.

\section{BALANCE MEASURE}

Subjects performed the One-Legged Stance Test (OLST), in which they stood on one leg with eyes closed as long as possible for up to 60 s per trial (Briggs et al., 1989). The test was repeated alternately on both legs three times. The average of the best time on each leg was chosen a priori as the outcome measure.

\section{COGNITIVE MEASURES}

Participants performed three CANTAB ${ }^{\circledR}$ (Cambridge) computer button box- and touchscreen-based tests [CANTABeclipse(TM), 2012]. In the SWM test participants search for a token in a group of up to eight boxes without returning to boxes where a token had been found in a previous trial. In the Stop Signal Task (SST) subjects are instructed to rapidly press a left or right button depending on the direction of a stimulus arrow. On a subset of trials, an auditory stop signal indicates that the subject must inhibit their response. The presentation time of the stop signal is adjusted over the course of the test so the participant is able to withhold a button press in half of the stop trials. In the Affective Go/No-Go test (AGN) the participant is informed of both the target and distractor valence (positive, negative, or neutral) for a rapidly presented series of words and instructed to press a button for words of the target valence only. Outcome measures identified a priori were SWM "between errors" (primary outcome measure: number of times the subject revisited a box in which a token was previously found, i.e., "between" trials with the same pattern of boxes), SST stop signal RT (a response inhibition measure: mean go-trial RT minus mean stop signal presentation time, so lower values are better), and AGN correct RT (average over positive, neutral, and negative valenced words). Additional measures included affective bias (AGN correct RT, positive minus negative valenced words), AGN RT variability (mean over three valences of SD of correct response RT), and SST RT variability (SD of RT on go trials). 


\section{SELF-REPORT}

Participants responded to four questionnaires: (1) Demographics: date of birth, sex, primary language, academic year and major, ethnicity, and race (first session only). (2) Adult ADHD Self-Report Scale (ASRS): 18 DSM-IV criteria as self-report Likert scale (0-4) items (Kessler et al., 2005). Of a priori interest was the sum score (0-24) from the six-question ASRS short screen consisting of inattention items $4,5,6$, and 9 and hyperactivity-impulsivity items 1 and 5. In exploratory analysis, the two sub-scores $(0-$ 36) were evaluated for all nine inattention items and all nine hyperactivity-impulsivity items. (3) Experience with Mind-Body Practices: mindfulness, meditation, yoga, etc. (4) Godin LeisureTime Exercise Questionnaire: frequency of strenuous, moderate, and mild exercise expressed as Weekly Leisure Activty Score (Godin and Shephard, 1985). In the Fall, subjects responded to paper versions of the questionnaires, while in the Spring, they responded to computer versions (Qualtrics). Additionally, the tai chi students were given a paper practice log and asked to keep a daily record of time spent practicing tai chi including class time; the log was collected at Session 2 and Session 3.

\section{STATISTICAL ANALYSIS}

Analyses were performed in R version 2.15.1 (R Development Core Team, 2012). Dependent variables were analyzed using a linear mixed effects model (LMER) with subject as a random effect and group and session as fixed effects. Effects of tai chi training were inferred from group $\times$ session interactions. Nuisance variables of age, sex, ESL, mind-body experience, and weekly leisure activity score were also included as fixed effects. Correlations between baseline scores and between change scores were evaluated by linear regression. Results with $p<0.05$ were considered significant, and no corrections were applied for multiple comparisons (five a priori measures and four exploratory measures).

\section{RESULTS}

These results are based on data from 28 tai chi students and 44 control subjects. The tai chi students reported that they trained
$101 \pm 24$ min per week. The effects of tai chi training were examined for the five quantities identified a priori, which provided specific measures of working memory, response inhibition, affective processing, physical balance, and the ADHD short screen. None of these measures exhibited significantly more change in the tai chi students compared to controls (Table 3). Changes from Session 1 to Session 3 in the affective go/no-go correct RT and the ADHD short screen were positively correlated across the tai chi students $[r(27)=0.511, p=0.006]$. There were no other correlations among changes in these five measures (remaining $p$ 's $>0.147$ ), nor among these five measures at session 1 (all p's $>0.225$ ).

Given the observed correlation in changes in the affective go/no-go correct RT and the ADHD short screen in the tai chi students, additional ADHD and affective go/no-go measures were explored (Table 4). As shown in Figure 1, inattention decreased by $10 \%$ relative to controls $(p=0.044$, group $\times$ session interaction, no group difference at baseline), but there was no significant change in hyperactivity-impulsivity $(p=0.748)$. Analysis of the less attentive subjects in each group (median split at session 1, no group difference at baseline, data not shown) yielded a $16 \%$ reduction in the tai chi students' report of inattention compared to that seen in controls $(p=0.002)$. In the small number of participants whose baseline inattention score was above 20, the optimum threshold for concordance with clinicianrated ADHD classification (Kessler et al., 2005), tai chi students' self-report of inattention declined by $22 \%$ compared to controls ( $p=0.023$, tai chi $n=3$, control $n=4$, no group difference at baseline, data not shown). Although the reduction in affective go/no-go RT variability was not significantly different between the two groups $(p=0.839)$, the numerical value of the affective go/no-go bias measure (positive-negative correct RT) increased in the tai chi students compared to controls $(p=0.008$ Figure 2).

Because RT variability has been proposed as a neurocognitive marker for ADHD (Tamm et al., 2012; Kofler et al., 2013), we explored correlations between the ADHD sub-scores for

Table 3 | Effect of tai chi training - measures specified a priori.

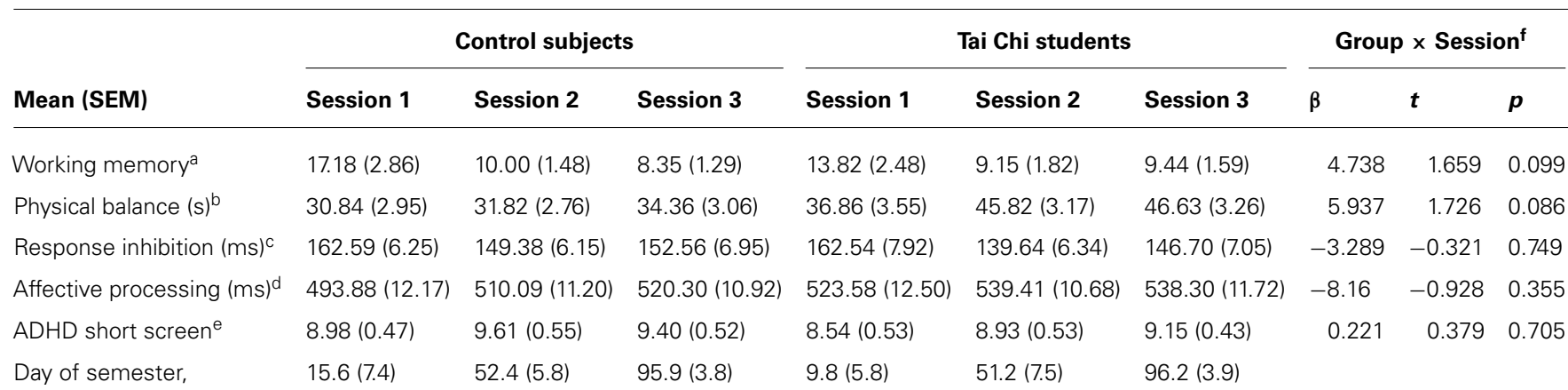

Mean (SD)

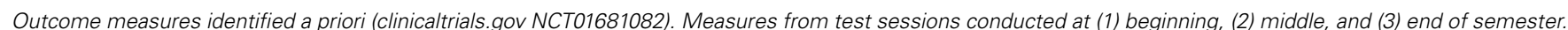

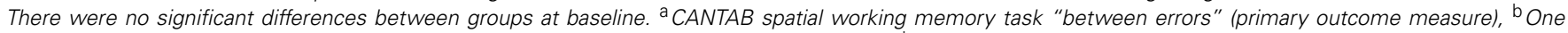

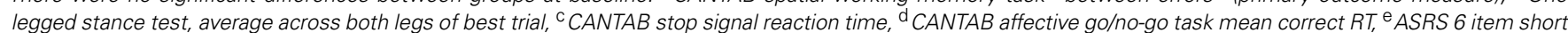

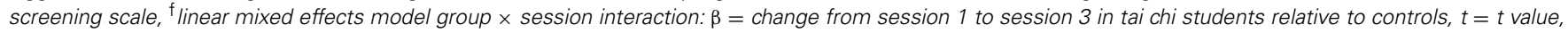
$p=2$-tailed $p$ value. 
Table 4 | Effect of tai chi training - ADHD and affective processing.

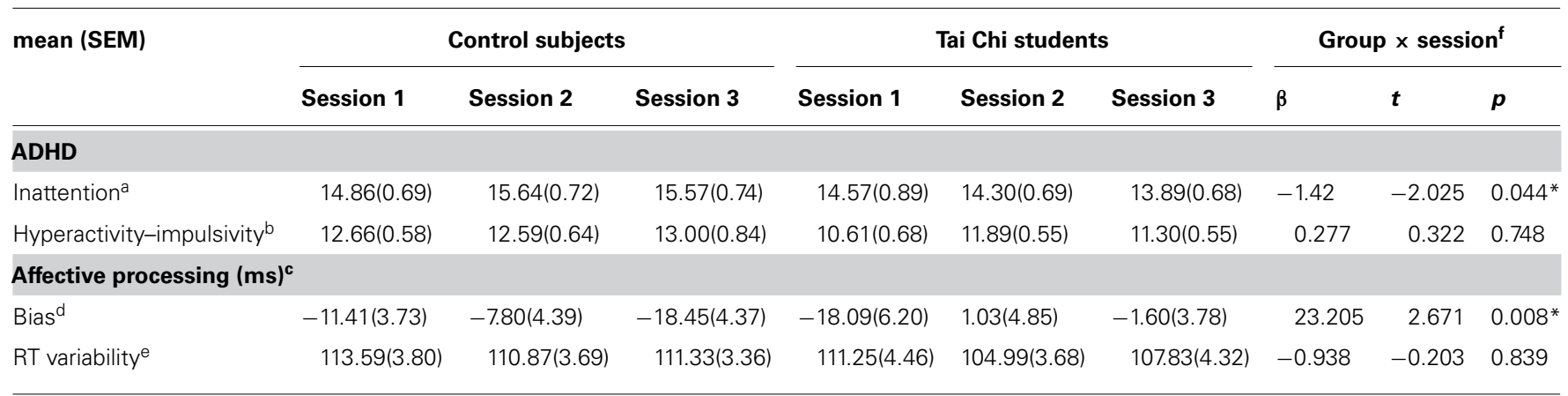

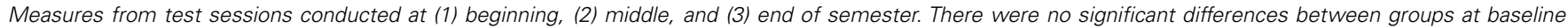

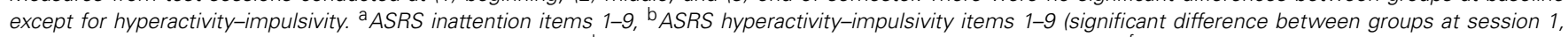

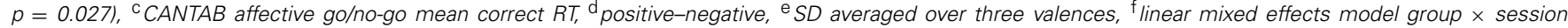
interaction: $\beta=$ change from session 1 to session 3 in tai chi students relative to controls, $t=t$ value, $p=2$-tailed $p$ value. ${ }^{*} p<0.05$.

inattention and for hyperactivity-impulsivity with RT variability in the SST and the affective go/no-go task (Table 5). As shown in Figure 3, at session 1, inattention correlated positively with affective go/no-go RT variability across all subjects $[r(72)=0.251$, $p=0.034]$. From session 1 to session 3, improvements in attention were correlated with reductions in affective go/nogo RT variability across the tai chi students $[r(27)=0.387$, $p=0.046]$ but not across the control subjects $[r(40)=0.073$, $p=0.655]$. There was, however, no significant group difference between these correlations $(p=0.20)$. In addition, tai chi students who reported more practice time tended to exhibit greater reductions in affective go/no-go RT variability at a trend level $[r(27)=-0.320, p=0.104]$. There were also correlations in unexpected directions of practice time with change in balance $[r(27)=-0.372, p=0.056]$ and change in the ADHD hyperactivity-impulsivity sub-score $[r(27]=0.397$, $p=0.040]$.

\section{DISCUSSION}

We examined the effects of tai chi training on selected attentional and cognitive processes in healthy young adults, and found a reduction in self-reported inattention that was supported by neurocognitive measures. These results suggest tai chi training might serve as therapy for young adults and adolescents suffering from ADHD inattention symptoms. Additional results pointed to the potential sensitivity of emotional processing measures to tai chi training.

Attention deficit hyperactivity disorder inattention indicators decreased in tai chi students, and the improvements in attention correlated with reductions in RT variability in an affective go/no-go task. Reviews of the literature suggest RT variability may be a marker of ADHD (Tamm et al., 2012; Kofler et al., 2013). Indeed, in addition to the correlated changes seen in the tai chi students, affective go/no-go RT variability correlated with inattention across all subjects at baseline. These improvements in attention observed in healthy young adults lend credence to the notion that tai chi training might serve as an effective therapy for adolescents and young adults with ADHD. Encouragingly, in less attentive subsets of subjects from each group, whose attention scores were equivalent at baseline, the tai chi students exhibited greater reductions in inattention compared to controls. However, while self-report of inattention decreased significantly compared to controls, there was no significant change in self-report of hyperactivity-impulsivity. To our knowledge, the only report of tai chi as therapy for ADHD describes a singlearm study of 13 adolescent patients, in which teacher report of symptoms declined after 5 weeks of training (Hernandez-Reif et al., 2001). The present results underscore the potential of tai chi training and indicate the need for additional studies in ADHD patients.

Beyond the observed reduction in self-report of inattention and associated reductions in affective go/no-go RT variability, the present study yielded additional interesting results. Affective bias increased in the tai chi students as the rates of response to positive and negative words tended to equalize. Among the measures identified a priori the only significant result was the correlation in changes in the ADHD six-question screen and affective go/no-go RT. This correlation was echoed by the correlation seen between the ADHD inattention score and the affective go/no-go RT variability. Contrary to our a priori hypotheses, we actually observed a trend-level decline in the tai chi students' performance of the spatial working memory task compared to controls, and no significant improvement was observed in the SST measure of response inhibition. Analyses of dose effect, i.e., correlations of change in outcome measures with practice time, present a complex picture and suggest the need for more accurate measures of time spent practicing tai chi. Physical balance improved in the tai chi subjects compared to the controls, but only at trend level. It is also noteworthy that in the stop signal task measure of response inhibition, correlations between ADHD measures and RT variability, though not significant, were in the same direction as those seen in the affective go/no-go task, i.e., reduced RT variability was associated with improved ADHD indicators. Taken together, these additional results suggest measures of affective processing may be sensitive to tai chi training.

These results contribute to a small but growing body of literature describing the effects of tai chi training in healthy young adults. This literature suggests that tai chi training may lead to improvements in self-report of physical and mental health 


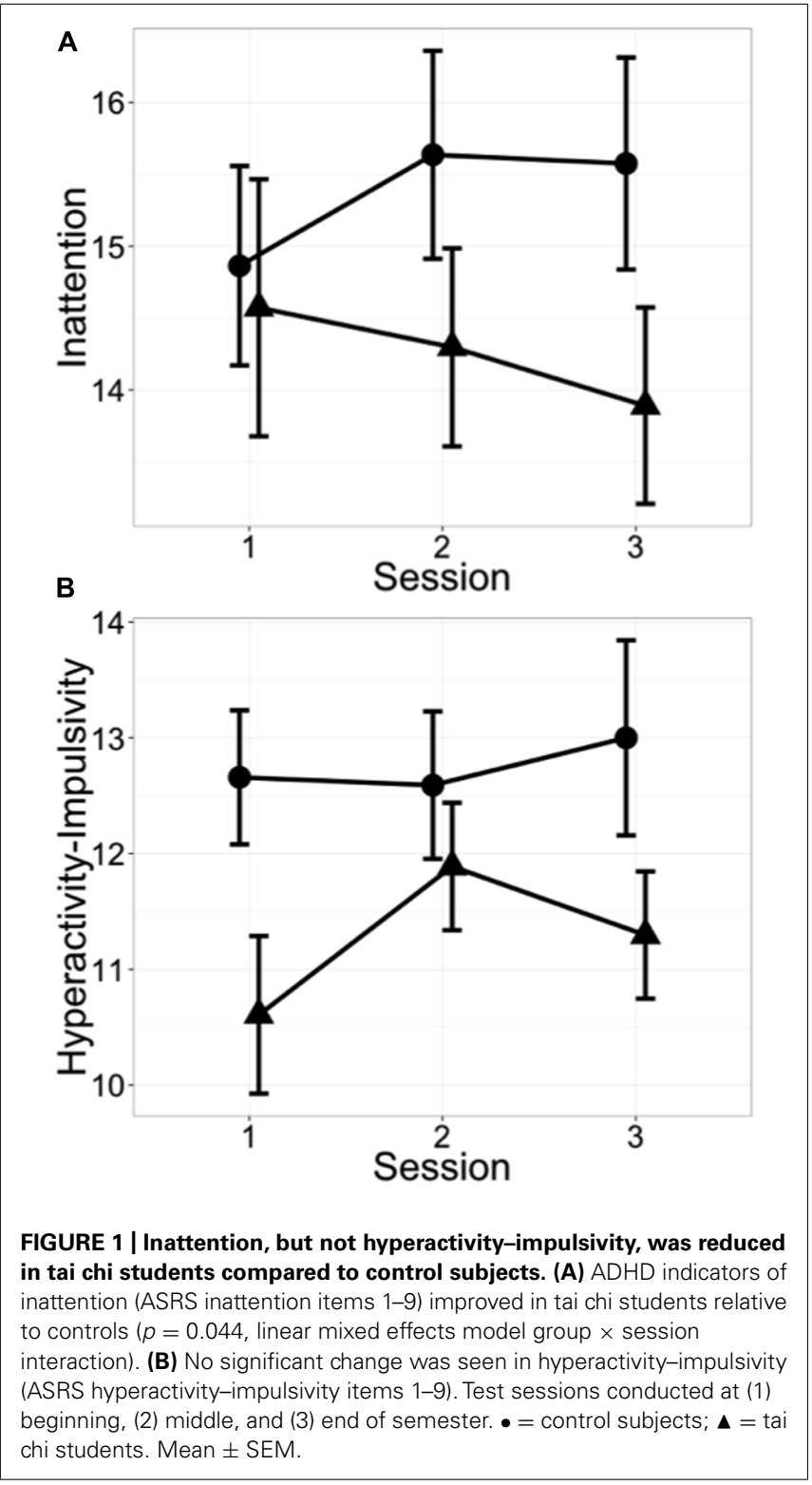

measures (Wang etal., 2004b), decreased nightmares (Slater and Hunt, 1997), improvements in self-report of mindfulness, mood, perceived stress, and sleep quality (Caldwell et al., 2011), reductions in salivary cortisol as well as improvements in selfreport of mental health measures and perceived stress (Esch et al., 2007), and improvements in measures of blood plasma immunological markers (Wang and An, 2011). Collectively, this literature suggests tai chi training in young adults may have salutary effects on mental health, perceived stress, and immune function. This literature also points to the need for more randomized controlled trials with objective measures of cognitive function.

The present study was strengthened by its interventional design, which, as opposed to a cross-sectional comparison of experienced and naive practitioners, permits inference of causality due to tai chi training. The use of a comparison group accounted

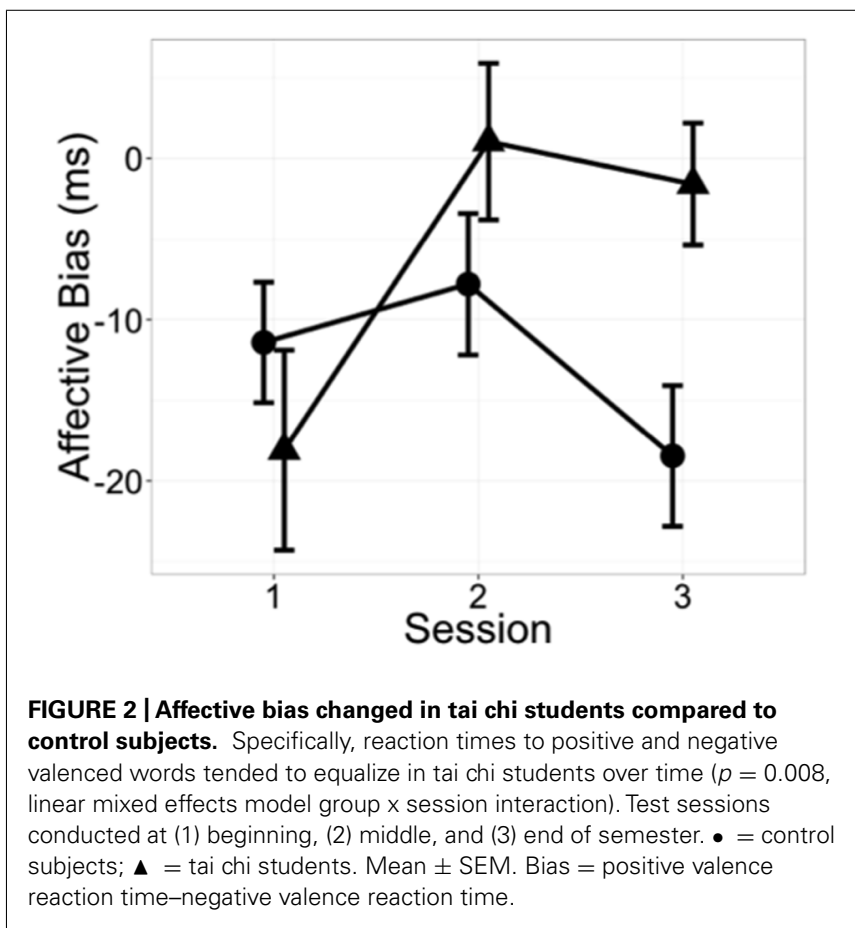

Table 5 | Correlations between ADHD subscores and reaction time variability: at baseline and change over time.

\begin{tabular}{|c|c|c|c|}
\hline Pearson $r$ & All subjects baseline ${ }^{g}$ & Control $\Delta^{\mathrm{h}}$ & Tai Chi $\Delta^{\mathrm{h}}$ \\
\hline \multicolumn{4}{|l|}{ Inattention ${ }^{a}$ vs } \\
\hline Stop signal task ${ }^{C}$ & 0.137 & 0.227 & 0.217 \\
\hline Affective go/no-go ${ }^{d}$ & $0.251 * \mathrm{e}$ & 0.073 & $0.387^{* f}$ \\
\hline \multicolumn{4}{|c|}{ Hyperactivity-impulsivity ${ }^{b}$ vs } \\
\hline Stop signal task & 0.077 & -0.026 & -0.175 \\
\hline Affective go/no-go & 0.108 & -0.058 & -0.104 \\
\hline $\mathrm{n}^{\mathrm{i}}$ & 72 & 40 & 27 \\
\hline
\end{tabular}

${ }^{a}$ ASRS inattention items 1-9; ${ }^{\mathrm{b}}$ ASRS hyperactivity-impulsivity items 1-9; ${ }^{C}$ CANTAB stop signal task, $S D$ of reaction time over go trials; ${ }^{d}$ CANTAB affective go/no-go task, correct RT SD averaged over 3 valences; ${ }^{e} p=0.034 ;{ }^{f} p=0.046$; $\mathrm{g}_{\text {correlation between measures at session } 1 ;}{ }^{\mathrm{h}}$ correlation between changes from session 1 to session 3; i session 3 data was unavailable for four control subjects and one tai chi student. ${ }^{*} p<0.05$.

for practice effects in testing. An additional strength was the use of objective neurocognitive measures. This study also had a number of limitations. The main findings resulted from exploratory analyses that do not survive multiple comparison correction, and they will require replication. It would be of particular interest to repeat the measures of inattention, RT variability, and affective bias. Although we used a control group, this was an observational study without randomization, so selfselection biases may exist. Mean age differed between the groups, although age was included as a nuisance variable in the linear mixed effects model. Because participants were aware that the purpose was to measure the psychological effects of tai chi training, demand characteristics may have influenced the 

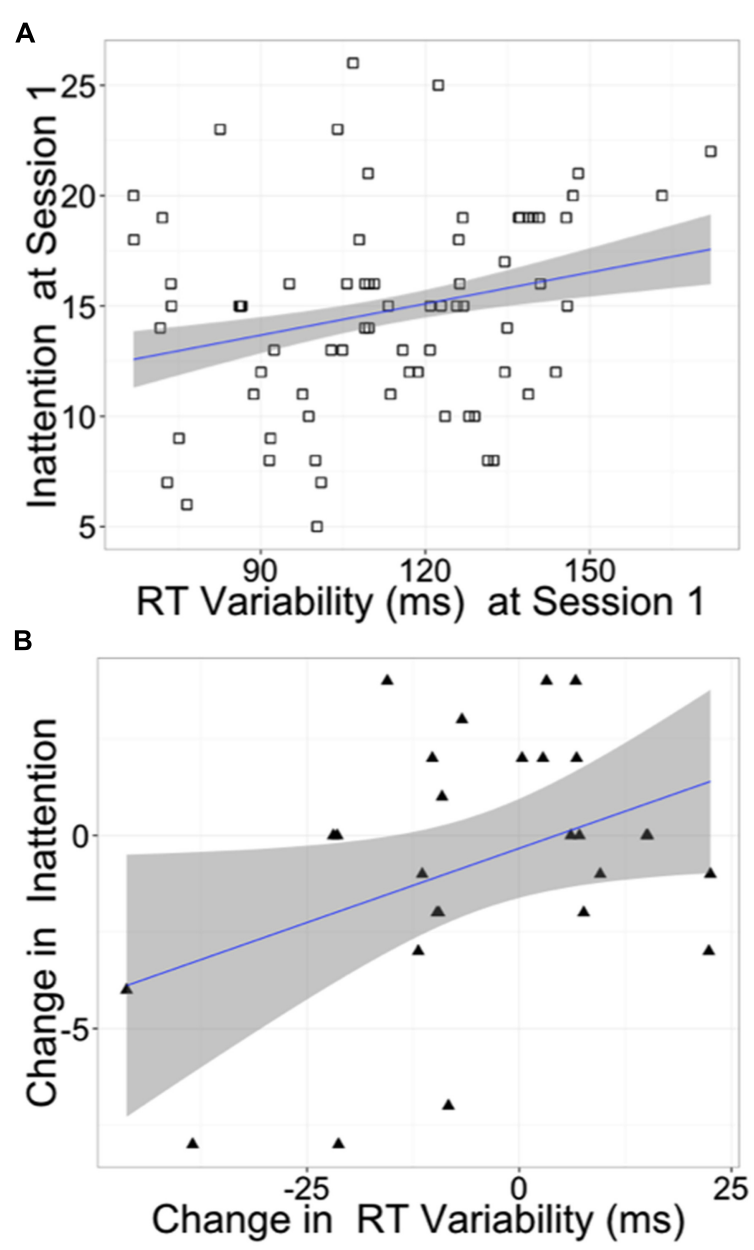

FIGURE 3 | Inattention and reaction time variability. (A) At baseline, inattention correlated with reaction time variability in the affective go/no-go task across all subjects $[r(72)=0.251, p=0.034]$, and (B) improvements in attention correlated with reductions in reaction time variability across the tai chi subjects $[r(27)=0.387, p=0.046]$. Change from session 1 to session 3. Inattention: ASRS inattention items 1-9. Reaction Time (RT) Variability: mean over three valences of affective go/no-go correct RT SD.

results, although the reduction in self-report of ADHD indicators was supported by the objective RT variability measures. Researchers were aware of the subjects' group status and may therefore have introduced bias in their administration of the tests. Because a single experienced teacher provided tai chi instruction, generalizability is limited. Finally, we did not assess potentially confounding medical or recreational drug use. Future studies of cognitive effects of tai chi training will ideally be randomized controlled trials with an active control intervention to balance the social and physical aspects of tai chi along with assessment of confounding variables including participant expectations.

In conclusion, the results of this study in healthy young adults suggest that tai chi training improves attention and may therefore hold potential as a non-pharmacological intervention for individuals with ADHD. Additional studies are needed to confirm these results in healthy subjects and to extend this research to ADHD patient populations.

\section{AUTHOR CONTRIBUTIONS}

Alexander K. Converse developed the study concept. Alexander K. Converse, Elizabeth O. Ahlers, and Richard J. Davidson contributed to the study design. Testing and data collection were performed by Alexander K. Converse. Alexander K. Converse and Brittany G. Travers performed the data analysis and interpretation. Alexander K. Converse drafted the paper. Brittany G. Travers and Richard J. Davidson provided critical revisions. All authors approved the final version of the paper for submission.

\section{ACKNOWLEDGMENTS}

Funding was provided by an anonymous donor. Additional support was provided by NIH grant P30 HD003352. The authors are grateful to Robert Yu for allowing us to recruit students in his tai chi course; to Lisa Flook, Robin Goldman, Daniel Levinson, and Stacey Schaefer for helpful discussions; and to Caitlin Bloomer, Clare Chandler, Erin Crain, Daniel Fehrenbach, Tyler Lieberthal, Malissa Scola, Chuanxin Wang, and Monica Whitehouse for data collection.

\section{REFERENCES}

American Psychiatric Association. (2000). Diagnostic and Statistical Manual of Mental Disorders: DSM-IV-TR, 4th Edn, Washington, DC: American Psychiatric Association.

Barnes, P. A. (2004). Complementary and alternative medicine use among adults: United States, 2002. CDC Adv. Data 343, 1-19.

Birdee, G. S., Wayne, P. M., Davis, R. B., Phillips, R. S., and Yeh, G. Y. (2009). T’ai Chi and Qigong for health: patterns of use in the United States. J. Altern. Complement. Med. 15, 969-973. doi: 10.1089/acm.2009.0174

Briggs, R. C., Gossman, M. R., Birch, R., Drews, J. E., and Shaddeau, S. A. (1989). Balance performance among noninstitutionalized elderly women. Phys. Ther. 69, 748-756.

Caldwell, K., Emery, L., Harrison, M., and Greeson, J. (2011). Changes in mindfulness, well-being, and sleep quality in college students through taijiquan courses: a cohort control study. J. Altern. Complement. Med. 17, 931-938. doi: 10.1089/acm.2010.0645

CANTABeclipse(TM), (2012). Test Administration Guide. Cambridge: Cambridge Cognition Ltd.

Castells, X., Ramos-Quiroga, J. A., Bosch, R., Nogueira, M., and Casas, M. (2011). Amphetamines for attention deficit hyperactivity disorder (ADHD) in adults. Cochrane Database Syst. Rev. 6, CD007813. doi: 10.1002/14651858.CD007813.pub2

Chamberlain, S. R., Robbins, T. W., Winder-Rhodes, S., Müller, U., Sahakian, B. J., Blackwell, A. D., et al. (2011). Translational approaches to frontostriatal dysfunction in attention-deficit/hyperactivity disorder using a computerized neuropsychological battery. Biol. Psychiatry 69, 1192-1203. doi: 10.1016/j.biopsych.2010.08.019

Durston, S. (2010). Imaging genetics in ADHD. Neuroimage 53, 832-838. doi: 10.1016/j.neuroimage.2010.02.071

Esch, T., Duckstein, J., Welke, J., and Braun, V. (2007). Mind/body techniques for physiological and psychological stress reduction: stress management via Tai Chi training - a pilot study. Med. Sci. Monit. 13, CR488-CR497.

Godin, G., and Shephard, R. J. (1985). A simple method to assess exercise behavior in the community. Can. J. Appl. Sport Sci. 10, 141-146.

Green, A. L., and Rabiner, D. L. (2012). What do we really know about ADHD in college students? Neurotherapeutics 9, 559-568. doi: 10.1007/s13311-012-0127-8 Hernandez-Reif, M., Field, T. M., and Thimas, E. (2001). Attention deficit hyperactivity disorder: benefits from tai chi. J. Bodyw. Mov. Ther. 5, 120-123. doi: 10.1054/jbmt.2000.0219

Jou, T. H. (1980). The Tao of Tai-Chi Chuan. Rutland, VT: Charles E. Tuttle. 
Kauz, H. (1974). Tai Chi Handbook: Exercise, Meditation, and Self-defense. Garden City, NY: Doubleday.

Kessler, R. C., Adler, L., Ames, M., Demler, O., Faraone, S., Hiripi, E., et al. (2005) The World Health Organization Adult ADHD Self-Report Scale (ASRS): a short screening scale for use in the general population. Psychol. Med. 35, 245-256. doi: $10.1017 /$ S0033291704002892

Knouse, L. E., and Safren, S. A. (2010). Current status of cognitive behavioral therapy for adult attention-deficit hyperactivity disorder. Psychiatr. Clin. North Am. 33, 497-509. doi: 10.1016/j.psc.2010.04.001

Kofler, M. J., Rapport, M. D., Sarver, D. E., Raiker, J. S., Orban, S. A., Friedman, L. M., et al. (2013). Reaction time variability in ADHD: a meta-analytic review of 319 studies. Clin. Psychol. Rev. 33, 795-811. doi: 10.1016/j.cpr.2013.06.001

Kooij, S. J. J., Bejerot, S., Blackwell, A., Caci, H., Casas-Brugué, M., Carpentier, P. J., et al. (2010). European consensus statement on diagnosis and treatment of adult ADHD: The European Network Adult ADHD. BMC Psychiatry 10:67. doi: 10.1186/1471-244X-10-67

Krisanaprakornkit, T., Ngamjarus, C., Witoonchart, C., and Piyavhatkul, N. (2010). Meditation therapies for attention-deficit/hyperactivity disorder (ADHD). Cochrane database Syst. Rev. 6, CD006507. doi: 10.1002/14651858.CD006507.pub2

Lam, L. C. W., Chau, R. C. M., Wong, B. M. L., Fung, A. W. T., Tam, C. W. C., Leung, G. T. Y., et al. (2012). A 1-year randomized controlled trial comparing mind body exercise (Tai Chi) with stretching and toning exercise on cognitive function in older Chinese adults at risk of cognitive decline. J. Am. Med. Dir. Assoc. 13, 568.e15-568.e20. doi: 10.1016/j.jamda.2012.03.008

Lara, C., Fayyad, J., de Graaf, R., Kessler, R. C., Aguilar-Gaxiola, S., Angermeyer, M., et al. (2009). Childhood predictors of adult attention-deficit/hyperactivity disorder: results from the World Health Organization World Mental Health Survey Initiative. Biol. Psychiatry 65, 46-54. doi: 10.1016/j.biopsych.2008.10.005

Levy, F. (1991). The dopamine theory of attention deficit hyperactivity disorder (ADHD). Aust. N. Z. J. Psychiatry 25, 277-283. doi: 10.3109/00048679109077746

Lu, X., Siu, K.-C., Fu, S. N., Hui-Chan, C. W. Y., and Tsang, W. W. N. (2013). Tai Chi practitioners have better postural control and selective attention in stepping down with and without a concurrent auditory response task. Eur. J. Appl. Physiol. 113, 1939-1945. doi: 10.1007/s00421-013-2624-9

Malmberg, K., Edbom, T., Wargelius, H.-L., and Larsson, J.-O. (2011). Psychiatric problems associated with subthreshold ADHD and disruptive behaviour diagnoses in teenagers. Acta Paediatr. 100, 1468-1475. doi: 10.1111/j.16512227.2011.02363.x

Man, D. W. K., Tsang, W. W. N., and Hui-Chan, C. W. Y. (2010). Do older t'ai chi practitioners have better attention and memory function? J. Altern. Complement. Med. 16, 1259-1264. doi: 10.1089/acm.2009.0462

Matthews, M. M., and Williams, H. G. (2008). Can Tai chi enhance cognitive vitality? A preliminary study of cognitive executive control in older adults after A Tai chi intervention. J. S. C. Med. Assoc. 104, 255-257.

Mortimer, J. A., Ding, D., Borenstein, A. R., DeCarli, C., Guo, Q., Wu, Y., et al. (2012). Changes in brain volume and cognition in a randomized trial of exercise and social interaction in a community-based sample of non-demented Chinese elders. J. Alzheimers Dis. 30, 757-766. doi: 10.3233/JAD-2012-120079

Nguyen, M. H., and Kruse, A. (2012). A randomized controlled trial of Tai chi for balance, sleep quality and cognitive performance in elderly Vietnamese. Clin. Interv. Aging 7, 185-190. doi: 10.2147/CIA.S32600

Qu, M. Y. (1999). Simplified Taijiquan (Revised Edition). Beijing: People's Sports Publishing House of China.

R Development Core Team. (2012). A Language and Environment for Statistical Computing. Vienna: R Foundation for Statistical Computing.
Slater, J., and Hunt, H. T. (1997). Postural-vestibular integration and forms of dreaming: a preliminary report on the effects of brief T'ai Chi Chuan training. Percept. Mot. Skills 85, 97-98. doi: 10.2466/pms.1997. 85.1.97

Sussman, S., Pentz, M. A., Spruijt-Metz, D., and Miller, T. (2006). Misuse of "study drugs:" prevalence, consequences, and implications for policy. Subst. Abuse Treat. Prev. Policy 1, 15. doi: 10.1186/1747-597X-1-15

Swanson, J. M., Kinsbourne, M., Nigg, J., Lanphear, B., Stefanatos, G. A., Volkow, N., et al. (2007). Etiologic subtypes of attention-deficit/hyperactivity disorder: brain imaging, molecular genetic and environmental factors and the dopamine hypothesis. Neuropsychol. Rev. 17, 39-59. doi: 10.1007/s11065-0079019-9

Tamm, L., Narad, M. E., Antonini, T. N., O’Brien, K. M., Hawk, L. W., Epstein, J. N. (2012). Reaction time variability in ADHD: a review. Neurotherapeutics 9, 500-508. doi: 10.1007/s13311-012-0138-5

Taylor-Piliae, R. E., Newell, K. A., Cherin, R., Lee, M. J., King, A. C., and Haskell, W. L. (2010). Effects of Tai Chi and Western exercise on physical and cognitive functioning in healthy community-dwelling older adults. J. Aging Phys. Act. 18, 261-279.

Toplak, M. E., Connors, L., Shuster, J., Knezevic, B., and Parks, S. (2008). Review of cognitive, cognitive-behavioral, and neural-based interventions for attentiondeficit/hyperactivity disorder (ADHD). Clin. Psychol. Rev. 28, 801-823. doi: 10.1016/j.cpr.2007.10.008

Wang, C., Collet, J. P., and Lau, J. (2004a). The effect of Tai Chi on health outcomes in patients with chronic conditions: a systematic review. Arch. Intern. Med. 164, 493-501. doi: 10.1001/archinte.164.5.493

Wang, Y. T., Taylor, L., Pearl, M., and Chang, L.-S. (2004b). Effects of Tai Chi exercise on physical and mental health of college students. Am. J. Chin. Med. 32, 453-459. doi: 10.1142/S0192415X04002107

Wang, M.-Y., and An, L.-G. (2011). Effects of 12 weeks' tai chi chuan practice on the immune function of female college students who lack physical exercise. Biol. Sport. 28, 45-9.6. doi: 10.5604/935875

Young, S., and Amarasinghe, J. M. (2010). Practitioner review: non-pharmacological treatments for ADHD: a lifespan approach. J. Child Psychol. Psychiatry 51, 116133. doi: 10.1111/j.1469-7610.2009.02191.x

Zylowska, L., Ackerman, D. L., Yang, M. H., Futrell, J. L., Horton, N. L., Hale, T. S., et al. (2008). Mindfulness meditation training in adults and adolescents with ADHD: a feasibility study. J. Atten. Disord. 11, 737-746. doi: $10.1177 / 1087054707308502$

Conflict of Interest Statement: The authors declare that the research was conducted in the absence of any commercial or financial relationships that could be construed as a potential conflict of interest.

Received: 04 November 2013; accepted: 07 January 2014; published online: 27 January 2014.

Citation: Converse AK, Ahlers EO, Travers BG and Davidson RJ (2014) Tai chi training reduces self-report of inattention in healthy young adults. Front. Hum. Neurosci. 8:13. doi: 10.3389/fnhum.2014.00013

This article was submitted to the journal Frontiers in Human Neuroscience.

Copyright (c) 2014 Converse, Ahlers, Travers and Davidson. This is an open-access article distributed under the terms of the Creative Commons Attribution License (CC BY). The use, distribution or reproduction in other forums is permitted, provided the original author(s) or licensor are credited and that the original publication in this journal is cited, in accordance with accepted academic practice. No use, distribution or reproduction is permitted which does not comply with these terms. 